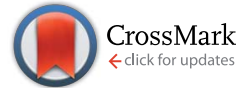

Cite this: J. Mater. Chem. A, 2016, 4, 7045

Received 28th February 2016

Accepted 16th March 2016

DOI: $10.1039 / c 6 t a 01770 c$

www.rsc.org/MaterialsA

\section{Plasmonic substrates comprising gold nanostars efficiently regenerate cofactor molecules $\uparrow$}

\author{
Ana Sánchez-Iglesias, ${ }^{a}$ Javier Barroso, ${ }^{b}$ Diego M. Solís, ${ }^{c} J_{0}$ M. M. Taboada, ${ }^{d}$ \\ Fernando Obelleiro, ${ }^{c}$ Valeri Pavlov, ${ }^{\text {b }}$ Andrey Chuvilin ${ }^{\text {ef }}$ and Marek Grzelczak ${ }^{\text {*af }}$
}

\begin{abstract}
The light harvesting capacity of plasmonic nanoparticles is a fundamental feature for catalysing chemical reactions close to their surface. The efficiency of the photochemical processes depends not only on the geometrical aspects on a single particle level but also on the complexity of the multiparticle architectures. Although, the effect of the particle geometry is progressively understood in the relevant photochemical processes (water splitting and hydrogen evolution), there are experimental and theoretical needs for understanding the role of the shape in the multiparticle systems in the photocatalytic processes. Here we have shown that macroscopic plasmonic substrates comprising gold nanostars exhibit better efficiencies than nanorods or cubes in the photoregeneration of cofactor molecules. We performed photochemical and photoelectrochemical measurements, supported by theoretical simulations, showing that the unique geometry of nanostars - radially distributed spikes contributes to stronger light absorption by the plasmonic film containing that type of nanoparticles.
\end{abstract}

\section{Introduction}

The unique properties of surface plasmon resonance ensure the consolidation of noble metal nanoparticles in photochemistry, a field of research and technology commanded currently by semiconductors. ${ }^{1-15}$ Some of the photochemical processes have been proposed recently in which plasmonic nanoparticles autonomously catalyse chemical reactions, such as water oxidation, ${ }^{16}$ hydrogen evolution, ${ }^{17}$ Suzuki coupling, ${ }^{18,19}$ alcohol oxidation $^{20,21}$ or reduction of nitroaromatic compounds. ${ }^{22}$

In a conventional photocatalytic system based on semiconductors, the performance in the catalytic process depends on the band gap energy of the material. In plasmon-based photochemistry, the photocatalytic capacity of particles is ruled by the energy of the surface plasmons, which is in turn related to the shape and size of the nanoparticles. ${ }^{23}$ In general, the isotropic particles with a diameter below $20 \mathrm{~nm}$ in the colloidal

\footnotetext{
aBionanoplasmonics Laboratory, CIC biomaGUNE, Paseo de Miramón 182, 20009 Donostia-San Sebastián, Spain. E-mail: mgrzelczak@cicbiomagune.es

${ }^{b}$ Biosensing Laboratory, CIC biomaGUNE, Paseo de Miramón 182, 20009 DonostiaSan Sebastián, Spain

'Departamento de Teoria de la Señal y Comunicaciones, University of Vigo, 36301 Vigo, Spain

${ }^{d}$ Departamento de Tecnología de los Computadores y de las Comunicaciones, University of Extremadura, 10003 Cáceres, Spain

${ }^{e}$ CIC nanoGUNE, Av. De Tolosa 76, 20018 Donostia-San Sebastián, Spain

IIkerbasque, Basque Foundation for Science, 48013 Bilbao, Spain

$\dagger$ Electronic supplementary information (ESI) available: TEM images, size analysis of the nanoparticles, control experiments for photoelectrochemical measurements, and SEM images. See DOI: 10.1039/c6ta01770c
}

phase absorb light in the visible spectral range (400-600 nm) while anisotropic particles, of similar volume, absorb/scatter light in the infrared spectral range $(700-1200 \mathrm{~nm})$. The optical features of the particles can be further altered in a multiparticle scenario, in which strong plasmon coupling enables intense electric field enhancement, which benefits light-mater interactions. Thus, the spectroscopic sensitivity to geometry on single- and multi-particle levels can be exploited to construct devices that absorb across the full solar spectrum. ${ }^{24,25}$

According to recent studies, plasmonic nanoparticles can increase the rate of chemical reactions by multiple mechanisms including the photo-thermal effect, electric near-field enhancement, and generation of hot electrons. ${ }^{11,15}$ In all of these mechanistic pathways, the particle shape plays an important role. Long et al. ${ }^{26}$ demonstrated that the plasmonic Pd concave nanostructures exhibit superior catalytic performance than cubes or octahedra. They ascribed such behavior to the photothermal effect and local heat at the reaction sites. Trinh et al. ${ }^{19}$ showed that plasmonic Pd hexagons are better than, again, cubes and octahedra in plasmon-assisted Suzuki coupling, driven by the hot electron pathway. From a broader perspective, it becomes straightforward that the photocatalytic efficiency of plasmonic nanoparticles increases with a decrease in the symmetry and the volume of the nanocrystal. ${ }^{27-29}$ For example, gold nanorods exhibit better photocatalytic properties than isotropic nanoparticles (spheres, cubes or plates), which is due to the strong enhancement of the field inside the nanocrystal. ${ }^{30}$ Thus, inspired by recent theoretical and experimental developments, we expect that the branched nanoparticles can bring a significant contribution to the plasmon-assisted 
photochemistry, in a similar manner to their contribution to the field of surfaced enhanced spectroscopy. ${ }^{31}$ The presence of multiple and non-evenly distributed spikes in the gold nanostars can improve light harvesting that in turn alters the enhancement of electric near-field and the generation of energetic charge carriers. ${ }^{32}$

The presence of co-catalyst on the particle surface can additionally improve the rate of the desired reaction. While bare plasmonic particles may exhibit negligible catalytic activity it can be improved in the presence of a surface metal, such as $\mathrm{Pt}^{33}$ $\mathrm{Pd},{ }^{34} \mathrm{Co},{ }^{16}$ or Ni. ${ }^{35}$ These surface metals facilitate binding events of the molecules that undergo chemical conversion, thereby decreasing the kinetic energy required to run the reaction. The spatial distribution of the co-catalyst on the photocatalyst metal is also an important factor. Weng et al. ${ }^{36}$ have shown that noncentrosymmetric architecture in the CdSe-Au-Pt hybrid system improves hot electron transfer from $\mathrm{Au}$ and Pt subunits, minimising their thermalisation. Thus, finding the most optimal shape and surface properties of the plasmonic nanoparticles is currently a key task in the strongly progressing field of all-metal photochemistry.

The present work focuses on the comparative study of the shape effect of the gold nanoparticles on the photoregeneration of the coenzyme molecules (nicotinamide adenine dinucleotide - NADH) using triethanolamine (TEAOH) as a sacrificial agent. We have recently shown that Pt-decorated gold nanorods can regenerate NADH when irradiated with visible or infrared light. ${ }^{33}$ Here, we tested cubes, rods and star-like nanoparticles in photochemical and photoelectrochemical experiments, showing that gold nanostars are the most catalytically active. We attribute such behaviour to the greater capacity of light harvesting by nanostars in aggregated state - plasmonic substrates - as compared to rods or cubes. Numerical simulations of the multiparticle system confirmed experimental evidence.

\section{Fabrication of plasmonic substrates containing particles of different shapes}

Prior to the synthesis of the particles we assumed that the starlike geometry consists of isotropic cores and one-dimensional spikes pointing outwards. Thus, to perform a comparative photochemical study as precise as possible, we decided to synthesise cube-like nanoparticles with dimensions similar to that of nanostar cores, and nanorods with dimensions similar to that of nanostar spikes. We chose a seed-mediated growth approach to obtain nanoparticles of three desired shapes: cubic, $^{37}$ rodlike, $^{38}$ and star-like ${ }^{39}$ (Fig. 1). For gold nanocubes, the mean value of the edge was equal to $31.9 \pm 1.9 \mathrm{~nm}$. For gold nanorods, the average width and length were $45.2 \pm 5.1$ and $10.9 \pm 1.0 \mathrm{~nm}$, respectively (aspect ratio $=4.1$ ). In the case of nanostars, the core diameter was $39.9 \pm 5.9 \mathrm{~nm}$. Regarding the spikes, the length and width at the base were $41.8 \pm 9.6$ and 13.1 $\pm 2.8 \mathrm{~nm}$, respectively. Detailed geometrical characterization and TEM images of the nanoparticles are provided in the ESI (see Fig. S1 and S2 $\uparrow$ ). The as-prepared particles were stabilised
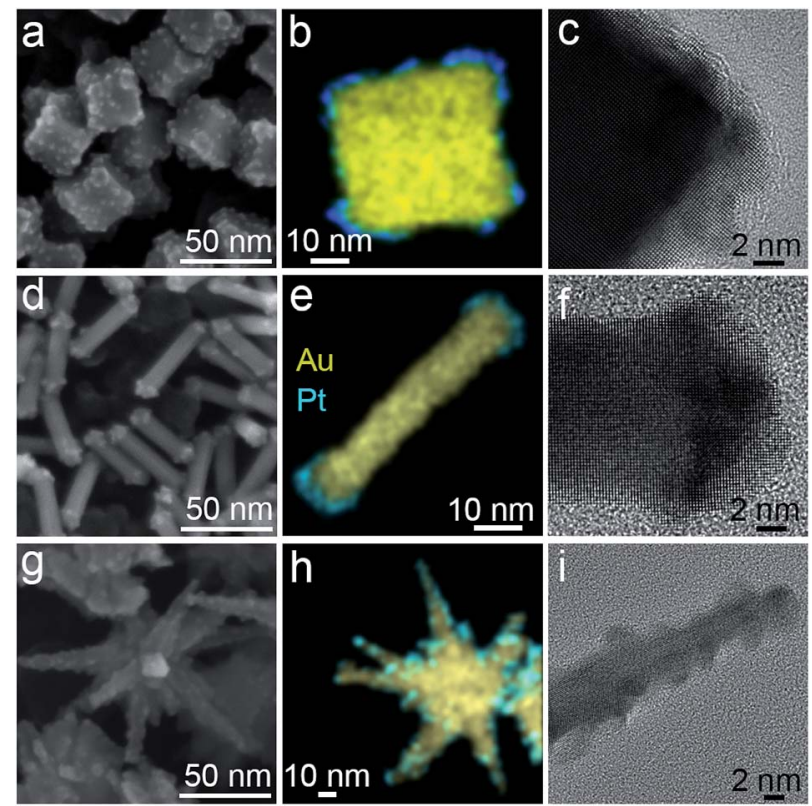

Fig. 1 Electron microscopy analysis of Pt-coated gold nanoparticles with different shapes: cube $(a-c)$, rod $(d-f)$ and star $(g-i)$. SEM images ( $a, d$, and $g$ ) and EDX mapping ( $b, e$, and $h$ ) confirming the presence of Pt on the gold surface. ( $c, f, i)$ HRTEM images of the nanoparticles showing epitaxial overgrowth of the Pt domains. All particles were covered with $10 \%$ mol of Pt.

with a cationic surfactant (cethyltrimethylammonium bromide - CTAB) in water. Since the presence of Pt is essential in the photochemical reaction, ${ }^{33}$ we subjected the as-prepared particles toward the overgrowth with Pt. The reduction of Pt took place in the presence of $\mathrm{Ag}^{+}$ions that ensured the formation of small domains, partially covering the surface. In the absence of silver, the platinum reduces in the form of a compact shell hindering the interaction of light with gold and thus decreasing the photochemical performance..$^{33,35}$

The analysis by Scanning Electron Microscopy (SEM) confirmed the presence of $\mathrm{Pt}$ on Au particles. As shown in Fig. 1a, d and g, the presence of bright spots on the particle surface correspond to Pt domains. The EDX mapping on the individual particle further revealed that $\mathrm{Pt}$ domains distribute mostly on the edges of cubes and stars, while in the case of gold nanorods, Pt locates only on the tips. Such a difference in spatial distribution relates to the crystalline structure of the initial gold nanoparticles. Single-crystalline gold nanorods exhibit higher surface anisotropy that allows Pt deposition only on the tips. The lateral part of the rods is blocked by silver-based species. ${ }^{40}$ Although cubes are also single-crystalline, the surface energy is rather isotropic - equally covered by $\{100\}$ facets. Thus, the Pt reduction takes places simultaneously on the edges and the flat parts. In the case of nanostars, the Pt reduction takes place on the whole spike surface. Although the star tips are highly anisotropic, the twinned planes (visible on HRTEM images) serve as nucleation points for $\mathrm{Pt}^{41}{ }^{41}$ leading to the homogenous distribution of co-catalyst domains. 
The HRTEM analysis revealed that Pt domains are crystalline (Fig. 1c, f and i), thus, confirming the presence of tight heterojunctions between both metals (for more detailed characterisation see ESI, Fig. S3†). Importantly, the size of Pt domains was similar in all samples: $2.0 \pm 0.4 \mathrm{~nm}$ (nanostars), $2.4 \pm 0.5$ $\mathrm{nm}$ (nanocubes) and $2.8 \pm 0.5 \mathrm{~nm}$ (nanorods).

The UV-vis-NIR measurements of the colloidal solutions of bare and Pt-coated particles confirmed successful Pt reduction. Regardless of the initial shape of gold particles, the presence of platinum caused the redshift of localised surface plasmon bands (LSPB) that is due to the relative changes in the aspect ratio. For the gold nanocubes, the plasmon band shifted from 557 to $570 \mathrm{~nm}$ (Fig. 2a), while in the case of nanorods and nanostars the longitudinal plasmon bands redshifted by 57 and $36 \mathrm{~nm}$, respectively (Fig. $2 \mathrm{~b}$ and c). To further confirm such optical behavior we performed numerical simulations of the particles with different morphologies using water as a medium. We employed the methodology that implements accelerated calculations based on surface integral equations with the method of moments formulation and the multilevel fast multipole algorithm. ${ }^{42,43}$ The input parameters of individual particles were based on the TEM and SEM measurements. For nanocubes, Pt was located on the edges, while for nanorods, on the tips (Fig. $2 \mathrm{~d}$ and $\mathrm{e}-$ insets). In the case of nanostars, the Pt shell was placed on the entire surface of spikes (Fig. 2f - inset) (for detailed parameterisation of each particle see Fig. $S 4 \dagger$ ). The simulated spectra correlate well with experimental results, showing the expected redshift and dumping of the plasmon band, confirming the strong interaction between both metals. ${ }^{44,45}$
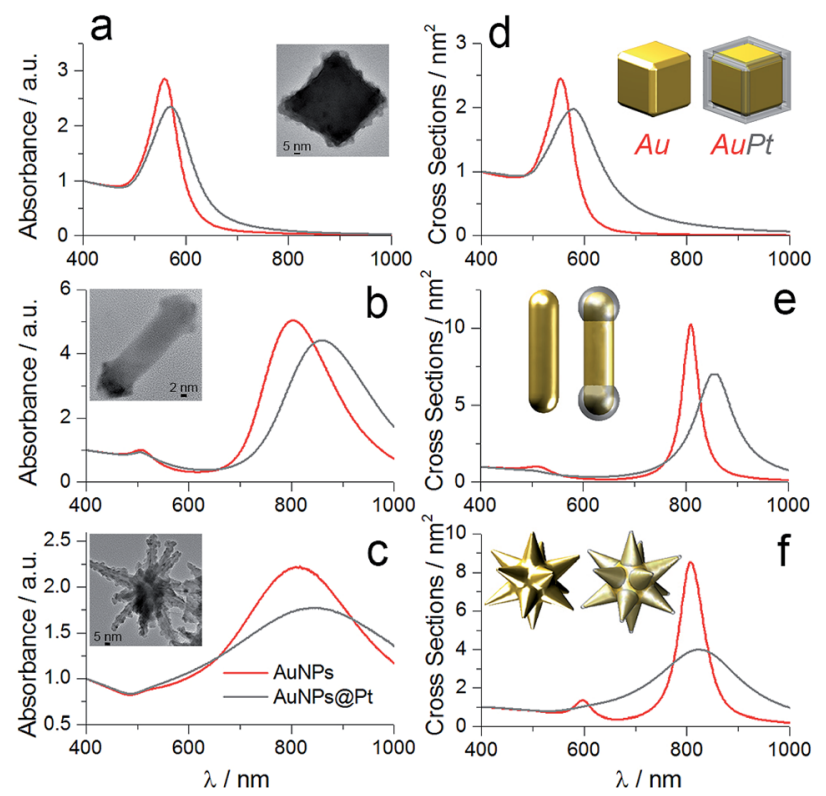

Fig. 2 Optical characterisation of the nanoparticles in solution. $(a-c)$ Experimental and $(\mathrm{d}-\mathrm{f})$ simulated spectra of the bare gold (red) and Ptcoated (grey) nanoparticles with cubic $(a, d)$, rod-like (b, e) and star-like morphology $(c, f)$. Insets in $(d-f)$ represent three-dimensional images of the particles used in simulations.

\section{Photochemistry and photoelectrochemical characterisation of plasmonic substrates}

Prior to photochemical characterisation, the particles were immobilised on the solid substrate (plasmonic substrate) ${ }^{33}$ Slow evaporation of the solution containing bare gold particles or Ptcoated on a glass substrate led to the formation of opaque films consisting of randomly distributed particles (Fig. S7 $\dagger$ ). In all cases we maintained a constant amount of metallic gold ( $5 \mathrm{mg}$ ).

Plasmonic substrates containing different particle shapes were subjected toward $\mathrm{NADH}$ photoregeneration. In a typical experiment, the substrate was immersed in a mixture $(1 \mathrm{~mL})$ containing $\mathrm{NAD}^{+}(1 \mathrm{mM})$, phosphate buffer $(\mathrm{pH}$ 8) and TEAOH $(1 \mathrm{M})$. The reduction of $\mathrm{NAD}^{+}$was initiated under light irradiation $\left(400-1200 \mathrm{~nm}, 150 \mathrm{~mW} \mathrm{~cm}{ }^{2}\right)$ at $35{ }^{\circ} \mathrm{C}$. The plasmonic substrates could be removed from the solution at any time for spectroscopic validation of the regeneration process (Fig. S5 $\dagger$ ). Since NADH exhibits a characteristic absorption band at $340 \mathrm{~nm}$ (molar absorption coefficient $6220 \mathrm{M}^{-1} \mathrm{~cm}^{-1}$ ), UV-vis spectroscopy provides a convenient qualitative control of the process. Overall, six substrates were evaluated, three containing bare gold nanoparticles and other three containing Pt-coated nanoparticles. Regardless of the particle shape, the regeneration of $\mathrm{NADH}$ was found to be $\sim 6$ times higher for Pt-coated particles as compared to the bare ones. Importantly, the best performing nanoparticles were gold nanostars both without and with Pt on the surface, suggesting that branched geometry is more efficient in the present process (Fig. 3).

Typically, the photoregeneration of NADH involves the transfer of $2 \mathrm{e}^{-}$and $\mathrm{H}^{+}$from TEAOH to $\mathrm{NADH}$, which is accomplished by simultaneous oxidation and reduction reactions. To ensure that the process takes place on the particle surface, we evaluated the interaction of TEAOH molecules with

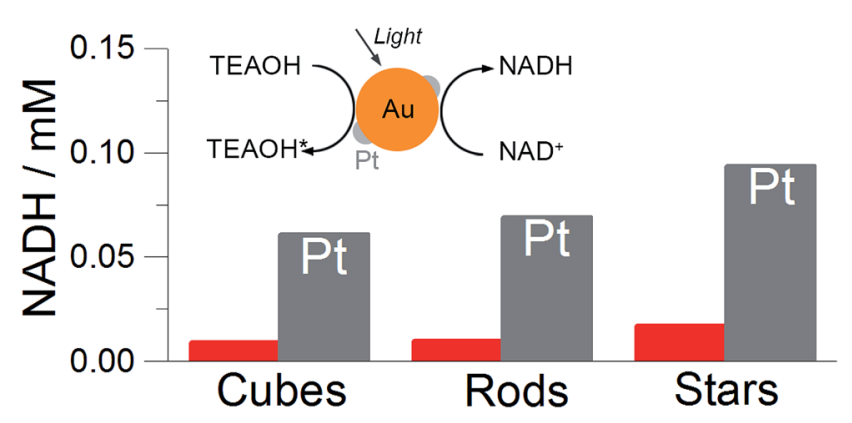

Fig. 3 Photochemical regeneration of cofactor molecules (NADH) under visible light irradiation on the plasmonic substrate containing different particles shapes. (inset) Scheme showing the reaction of simultaneous oxidation of $\mathrm{TEAOH}$ and reduction of $\mathrm{NAD}^{+}$to $\mathrm{NADH}$. The final concentration of NADH after a two hour reaction in the presence of plasmonic substrate containing different particle shapes. Red bars represented the concentration of the NADH when bare nanoparticles were used; the grey columns correspond to Pt-coated nanoparticles. In all experiments, the substrates were immersed in the solution containing TEAOH (1 M), NAD ${ }^{+}(1 \mathrm{mM})$ and phosphate buffer $(\mathrm{pH})$ and irradiated with light $\left(400-1200 \mathrm{~nm}, 150 \mathrm{~mW} \mathrm{~cm}{ }^{-2}\right)$ at $35^{\circ} \mathrm{C}$ for two hours. 
plasmonic nanoparticles by comparing the position of the maximum of LSPB in water and $0.1 \mathrm{M}$ TEAOH. The LSPB blue shifted $2 \mathrm{~nm}$ in the case of cubes and $3 \mathrm{~nm}$ for nanorods and stars (Fig. S6 $\dagger$ ). Such behavior is typical for the particles that are exposed to the electron-rich environment and proves the spontaneous electron injection from the molecules to particles. ${ }^{46}$ Although the Pt domains are crucial for the oxidation of TEAOH molecules, it is hard to assess to what extent the reduction of $\mathrm{NAD}^{+}$to $\mathrm{NADH}$ takes place on platinum. ${ }^{33}$ Since the photooxidation of TEAOH is a multistep process involving the formation of many intermediate species, ${ }^{47}$ we found it appropriate to perform separate photoelectrochemical analysis of the oxidation reaction.

Photoelectrochemical measurements confirmed the trends observed for different particle shapes in photochemical reactions. We performed photoelectrochemical studies of TEAOH oxidation on the electrode coated with nanoparticles. We used a three-electrode system (DropSens) consisting of a carbon working electrode, silver pseudo-reference, and carbon counter electrode. The solutions containing particles were drop-cast on the working electrodes and slowly evaporated under ambient conditions to maximise their homogenous distribution. Although we noticed the inevitable formation of coffee rings on the electrode edges, the surface coverage was similar in all samples containing particles of different shapes (Fig. S7 $\dagger$ ).

The electrode was covered with a $0.05 \mathrm{~mL}$ of the solution containing TEAOH (1 M) and phosphate buffer. To obtain the amperometric $i-t$ curve, the current was stabilised for $6 \mathrm{~min}$ at circuit potential $(\mathrm{ca} .-0.36 \mathrm{~V})$. Then the light was switched on for 10 seconds and an immediate increase in the current was observed (Fig. 4). We noticed that the presence of Pt on the gold surface was crucial to oxidise TEAOH. In the presence of Pt, the photocurrent was approximately ten times higher as compared to the photocurrent obtained from the bare gold nanoparticles regardless of their shape (red line in Fig. 4a, S8 and S9†). Such improvement is due to the low interfacial energy barrier between $\mathrm{Pt}$ and $\mathrm{Au}$ and suggests the contribution of the photoelectrocatalytic process. ${ }^{36}$

For all the particles coated with $\mathrm{Pt}$, the photocurrent consisted of two temporal components: a fast component that increased with time constant $\sim 0.5 \mathrm{~s}$ and an additional slow component that constantly increased with a time of $\sim 10 \mathrm{~s}$ (Fig. 4a). The origin of the "fast" components is the contribution of the photoelectrocatalytic process that can involve hot electron generation that immediately flow to the counter electrode. ${ }^{48}$ The "slow" components are a result of the photothermal effect of the illumination. ${ }^{49}$ Importantly, the magnitude of photocurrent, for both slow and fast components, depends on the particle shape and increases in the following order: cubes, rods, and stars, which is in good agreement with photochemical reactions (Fig. 3). The photocurrent of the nanostars added 250 $\mu \mathrm{A} \mathrm{cm} \mathrm{cm}^{-2}$ after $10 \mathrm{~s}$ of illumination, which is 3.5 and 1.5 times higher than that of nanocubes and nanorods, respectively. A similar trend has been observed for the "fast" component: the photocurrent of the nanostars was 2.5 and 1.5 times higher than that of the nanocubes and nanorods, respectively. SEM inspection confirmed morphological stability of the

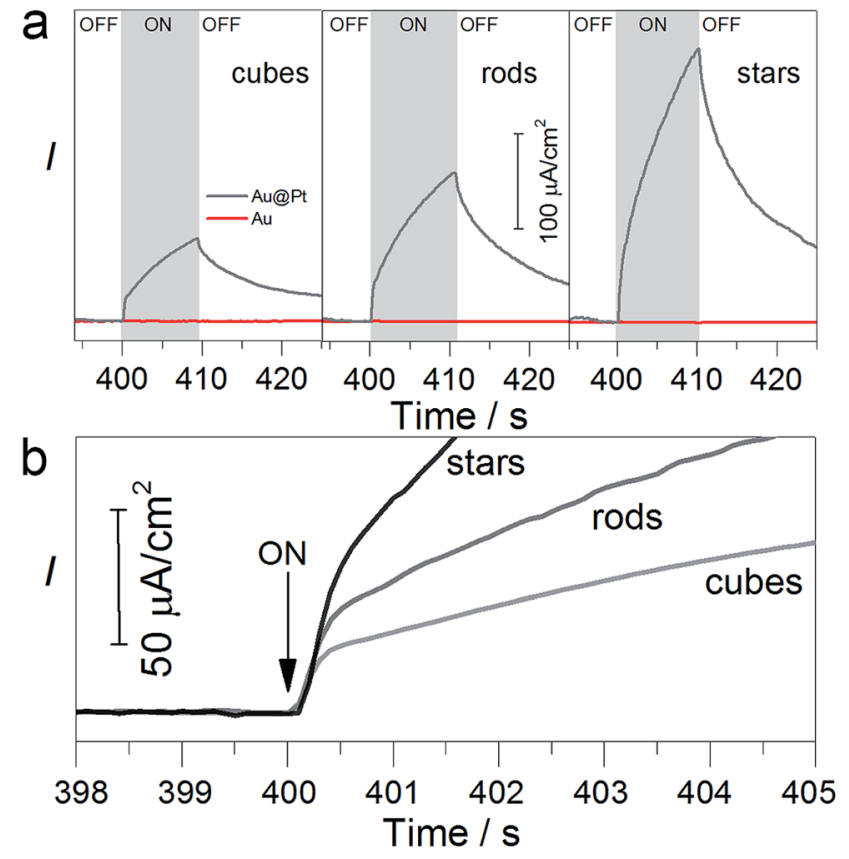

Fig. 4 Photoelectrochemical characterisation of TEAOH oxidation on the nanoparticles with different shapes. (a) Amperometric $i-t$ curves for bare cubes, rods and stars (red lines) and platinum-coated (grey lines). Photocurrent exhibits 'fast' (0.5 s) and 'slow' components (10 s). (b) Fast current component for three samples containing Pt-coated particles of different shapes showing better performance of stars than rods and cubes. In all experiments, the electrodes were immersed in the solution containing TEAOH (1 M), phosphate buffer $(\mathrm{pH} 8)$ and irradiated with light $\left(400-1200 \mathrm{~nm}, 150 \mathrm{~mW} \mathrm{~cm}{ }^{-2}\right)$ at $0 \mathrm{~V}$ at $25^{\circ} \mathrm{C}$. Each electrode was covered by the same amount of nanoparticles in terms of metallic gold.

nanoparticles (Fig. S10 $\dagger$ ). Overall, the photoelectrochemical measurements correlate with photochemical reactions, showing that the photoelectrocatalytic effect plays an important role and is strictly related to the particle shape.

Since the presence of Pt on the gold particle surface was crucial for achieving the photochemical reaction, we measured the relative amount of this metal for different plasmonic substrates. For estimation of the Pt/Au ratio we used XPS techniques (Fig. S11 $\dagger$ ), showing that stars and cubes contained a similar amount of surface platinum with respect to gold: $\mathrm{Pt} / \mathrm{Au}$ $=0.22$ (stars) and $\mathrm{Pt} / \mathrm{Au}=0.20$ (cubes). Gold nanorods contained less $\mathrm{Pt}(\mathrm{Pt} / \mathrm{Au}=0.09)$ that is due to the limited surface area accessible for Pt - tips. Relatively similar values of $\mathrm{Pt}$ and $\mathrm{Au}$ for different shapes suggest that the morphology of the initial particles is a key parameter that determines the distribution of the particles in the film and defines their interaction with incident light.

\section{Optical properties of plasmonic substrates - experimental and numerical characterisation}

Based on the experimental results we assume that better light absorption determines the superior photocatalytic performance 

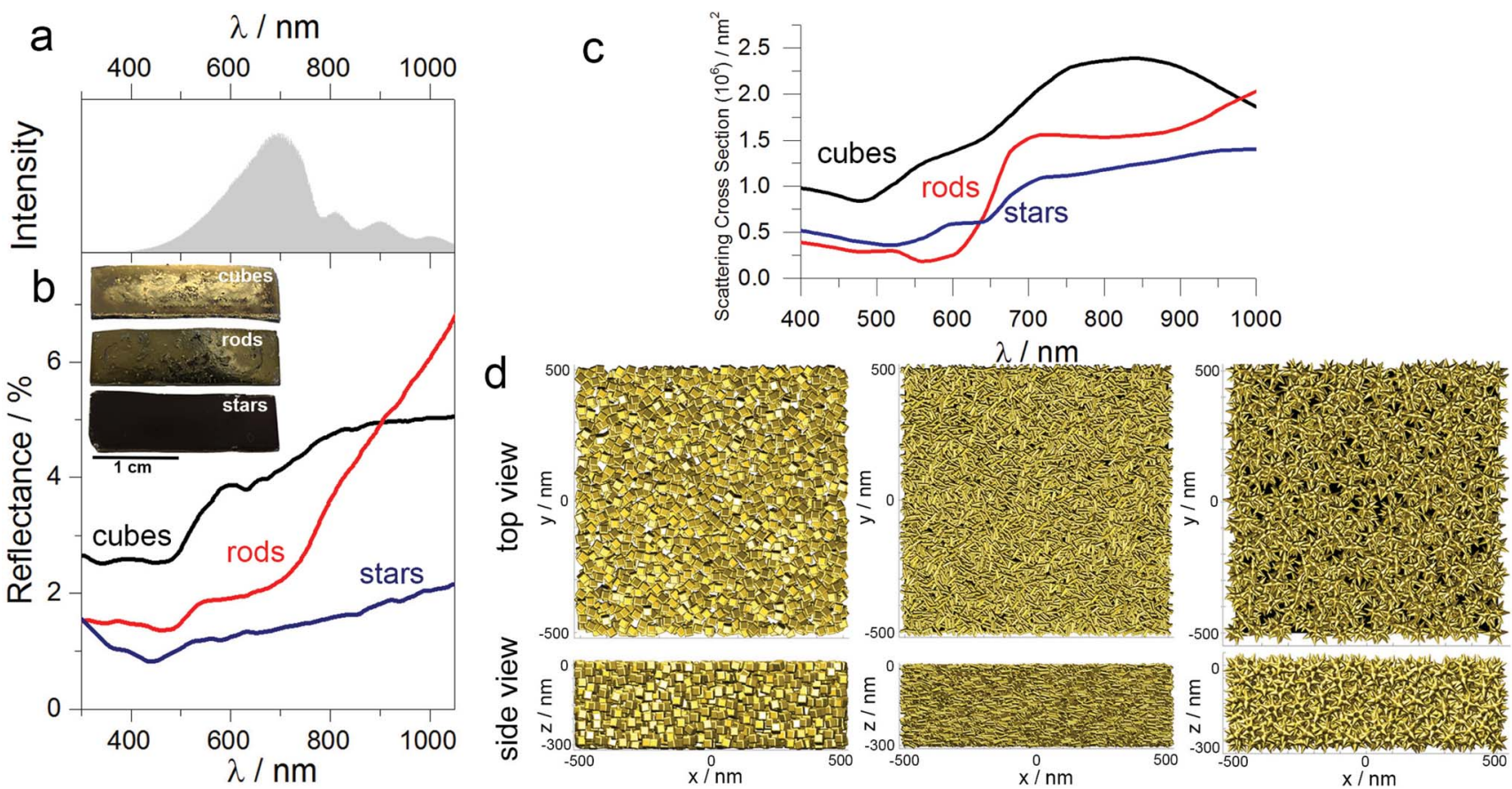

Fig. 5 Optical characterisation of the plasmonic substrate. (a) Spectral feature of the lamp used in all experiments. (b) Diffuse reflection spectra of substrates comprising cubes, rods and stars, showing better light harvesting capacity by star-containing plasmonic substrates. (inset) Images of the substrates comprising gold nanoparticles. (c) Simulated scattering cross-section spectra of the substrate comprising three different morphologies. (d) Top view (upper panel) and side view (lower panel) of modelled architectures used for spectra simulations in (c): nanoparticles with different geometries compacted in a $0.3 \mu \mathrm{m}^{3}$ box (minimum surface-to-surface separation is $1 \mathrm{~nm}$ ).

of the substrate containing nanostars, as compared to the nanorods or cubes. Visual inspection shows that the substrates containing cubes and rods are shinier than the substrates containing nanostars, which are practically black (see inset in Fig. 5b). Diffuse reflection measurements confirmed such observations, revealing that the substrates containing gold nanorods or cubes reflect more light in the spectral range above $500 \mathrm{~nm}$ (Fig. 5b). The difference in light reflection for all shapes is even more pronounced at the maximum of the intensity of the incident light $(c a .700 \mathrm{~nm})$ that we used in all experiments (Fig. 5a), additionally affecting the photochemical performance.

To further correlate our experimental results, we simulated the scattering cross-section spectra of substrates comprising nanoparticles with different shapes. Each model plasmonic substrate contained randomly packed particles: 2194 (cubes), 8071 (rods) and 501 (nanostars), filling a film-like box of 0.03 $\mu \mathrm{m}^{3}$ (Fig. 5d), with a minimum distance between the particle surface of $1 \mathrm{~nm}$. The calculated scattering spectra displayed typical broadening, suggesting multiple plasmon couplings, which are in good agreement with the experimental reflectance spectra (compare Fig. 5b and c). These results indicate that nanostars in the aggregated mode scatter less amount of light as compared to the nanocubes or nanorods. We hypothesise, then, that such an optical response is related to the intrinsic shape of the individual building blocks. During the drying process, the nanorods or cubes sediment in-parallel to the substrate surface, pointing sidewise toward the incident light, thereby increasing the contribution of the specular reflection. In the case of nanostars, however, radial distribution of spikes increases the diffuse reflection, and, therefore, minimise the amount of light that is bounced back to the medium. Such difference in optical properties in the aggregated state is especially visible in the case of gold nanorods and nanostars. Although the maximum of LSPB for both geometries in solution is very similar (Fig. 2), the reflectance above $800 \mathrm{~nm}$ is much larger in the case of nanorods than for stars, confirming more efficient light absorption by the plasmonic substrate containing nanostars.

It is important to stress, that spike-like, or conical shape is more appropriate for photochemical application than nanorods, or cylinders. Although, we cannot compare, at the present stage, the performance of spike only and nanorods, we can support our speculation by the recent work by Weng et al.,${ }^{36}$ who showed that nanoparticles that lack the central symmetry exhibit better performance due to the more efficient charge separation. Single spike located on the star core is a noncentrosymmetric object because of its unequal width on the tips. Therefore, we postulate that the unique geometry of the spike tips can render better photochemical conversion on nanostars than other shapes.

Finally, the unique geometry of nanostars - radial spike distribution - hinders close contact between the particles in the plasmonic film, facilitating the formation of the pores that improves the access of the molecules to the particle surface. Thus, the active surface area of the plasmonic substrate containing nanostars can be higher than that of the nanorods and cubes (Fig. S12†). 


\section{Conclusions}

In conclusion, we studied the shape effect of the platinum coated gold nanoparticles on the photoregeneration of cofactor molecules. In photochemical and photoelectrochemical measurements, we found that substrates comprising branched nanoparticles are better photocatalysts as compared to the rodor cube-like particles. We explain such behaviour by the geometrical features of gold nanostars that efficiently harvest the light improving photoelectrocatalytic effect. Future studies envisage the study of photochemical performance using nanostars with different spike lengths. This work opens up possibilities for efficient all-metal photochemistry and establish the basement for the design of plasmonic catalytic materials.

\section{Experimental}

\section{Materials}

Gold(III) chloride hydrate $\left(\mathrm{HAuCl}_{4}\right)$, sodium citrate tribasic dihydrate, hexadecyltrimethylammonium bromide (CTAB), hexadecyltrimethylammonium chloride (CTAC), silver nitrate $\left(\mathrm{AgNO}_{3}\right)$, hydrochloric acid $(\mathrm{HCl})$, ascorbic acid, sodium borohydride $\left(\mathrm{NaBH}_{4}\right)$ and potassium(II) tetrachloroplatinate $\left(\mathrm{K}_{2} \mathrm{PtCl}_{4}\right)$ were purchased from Sigma-Aldrich and were used without further purification.

\section{Characterisation}

Spectroscopy. UV-vis spectra were collected using a scanning spectrophotometer Agilent 8453 UV/vis diode-array spectrophotometer. Diffuse reflection spectra were recorded with a spectrophotometer Maya2000 Pro (Ocean Optics) equipped with a reflection probe (Ocean Optics, QR400-7-UV-BX) located in a $6.35 \mathrm{~mm}$ holder used as a reference diffuse reflectance standard (PTFE, WS-1). The illumination source was a $150 \mathrm{~W}$ quartz halogen fiber optic illuminator (Fiber-Lite ${ }^{\circledR} \mathrm{MI}-150^{\mathrm{TM}}$, Dolan-Jenner Industries). XPS experiments were performed in a SPECS Sage HR 100 spectrometer with a non-monochromatic $\mathrm{X}$-ray source (Magnesium $\mathrm{K} \alpha$ line of $1253.6 \mathrm{eV}$ energy and 250 $\mathrm{W})$. The samples were placed perpendicular to the analyser axis and calibrated using the $3 \mathrm{~d}_{5 / 2}$ line of $\mathrm{Ag}$ with a full width at half maximum (FWHM) of $1.1 \mathrm{eV}$. The selected resolution for the spectra was $15 \mathrm{eV}$ of pass energy and $0.15 \mathrm{eV}$ per step. All measurements were performed in an ultrahigh vacuum (UHV) chamber at a pressure around $5 \times 10^{-8}$ mbar.

Electron microscopy. (Scanning) Transmission electron microscopy ((S)TEM) images were collected with a JEOL JEM1400PLUS operating at $120 \mathrm{kV}$ and Titan 60-300 TEM/STEM (FEI, Netherlands) operating at $300 \mathrm{kV}$. EDX mapping was performed in scanning mode using Spectral Imaging (SI) capability. Scanning electron microscopy (SEM) images were acquired using a Dual Beam SEM/FIB Helios 450S microscope (FEI, Netherlands) at 5 and $10 \mathrm{kV}$ acceleration voltages.

Photoelectrochemistry. Electrochemical measurements were performed with an Autolab PGSTAT302N potentiostat controlled by the NOVA software. We used disposable screenprinted carbon electrodes (SPCES, DropSens) that consist of a three-electrode system supported on a ceramic substrate. The working electrode consists of carbon (4 mm diameter). Silver and carbon were used as the pseudo-reference and counter electrodes, respectively. All measurements were carried out at room temperature.

Optical modelling. Spectral simulations were carried out using a numerical method based on the surface integral equation-method of moments (SIE-MoM) formulation (M3 solver). ${ }^{\mathbf{4 2}}$ With this methodology, the metallic nanostructures are replaced by equivalent electric and magnetic currents placed over the particle boundary surfaces and interfaces. This brings about important advantages with respect to volumetric approaches, strongly reducing the computation domain as only the material boundaries and interfaces must be parameterized. Additionally, this methodology yields very accurate and stable solutions, particularly when dealing with resonant metallic responses, as the singular behavior of fields is analytically handled by the Green's function and its derivatives. Through Maxwell's equations and boundary conditions for the total fields, a set of SIEs is derived for the unknown surface equivalent currents. These SIEs are subsequently discretized by applying a Galerkin MoM procedure in terms of a set of basis and testing functions, leading to a dense $N \times N$ matrix system of linear equations ${ }^{50}$ ( $N$ being the number of basis functions used to expand the unknown equivalent currents).

For the realistic simulation of the large-scale plasmonic systems considered here, comprising assemblies of more than 8000 nanoparticles, SIE-MoM was expedited via the multilevel fast multipole algorithm-fast Fourier transform (MLFMAFFT), ${ }^{51,52}$ and combined with robust Schwarz domain decomposition preconditioning, ${ }^{53}$ resulting in a high algorithmic efficiency-computational cost of $\mathrm{O}(N \log N)$-along with highscalability via parallelization using multicore computer clusters.

The simulations were carried out on a workstation with four 16-core Intel Xeon E7-8867v3 45 MB Smart-Cache processors at $2.50 \mathrm{GHz}$. A high surface mesh density was considered leading to dense matrix systems of 5.7, 7.6 and 4.1 million unknowns for the substrates comprising cubes, rods and stars, respectively. A relative error norm of $10^{-5}$ was set to stop the Krylov iterative solver. Considering the above, the computation times per wavelength ranged from $402 \mathrm{~s}$ (at $400 \mathrm{~nm}$ ) to $2450 \mathrm{~s}$ (at $1000 \mathrm{~nm}$ ) for the cube substrate, 382 to $2057 \mathrm{~s}$ for the rod substrate, and 466 to $3138 \mathrm{~s}$ for the star substrate. The memory usage ranged from 27 to 93 GB for the cube substrate, 71 to 149 GB for the rod substrate, and 23.5 to $67 \mathrm{~GB}$ for the star substrate.

\section{Synthetic procedures}

Synthesis of gold nanocubes. The synthesis of gold cubes was carried out by a seeded growth process. ${ }^{37}$ To prepare gold seeds, gold salt $\mathrm{HAuCl}_{4}(0.5 \mathrm{mM})$ was reduced by $\mathrm{NaBH}_{4}$ $(0.3 \mathrm{mM})$ in the presence of CTAC $(5 \mathrm{~mL}, 100 \mathrm{mM})$. An aliquot of seed solution $(0.025 \mathrm{~mL})$ was added to a growth solution containing CTAC (50 mL, $100 \mathrm{mM}), \mathrm{HAuCl}_{4}(2.5 \mathrm{~mL}, 10 \mathrm{mM})$, $\mathrm{AgNO}_{3}(0.5 \mathrm{~mL}, 10 \mathrm{mM}), \mathrm{HCl}(1 \mathrm{~mL}, 1000 \mathrm{mM})$ and ascorbic acid 
(0.5 mL, $100 \mathrm{mM})$. The reaction was gently shaken after the addition of the seeds and left undisturbed overnight. The solution was centrifuged twice (5500 rpm, $30 \mathrm{~min}$ ) and redispersed in CTAB $(100 \mathrm{mM})$ to obtain a final concentration of gold equal to $0.5 \mathrm{mM}$.

Synthesis of gold nanorods. Gold nanorods were prepared using Ag-assisted seeded growth. ${ }^{38}$ Seeds were prepared by the reduction of $\mathrm{HAuCl}_{4}(5 \mathrm{~mL}, 0.25 \mathrm{mM})$ with $\mathrm{NaBH}_{4}(0.3 \mathrm{~mL}$, $10 \mathrm{mM})$ in aqueous CTAB solution $(100 \mathrm{mM})$. An aliquot of seed solution $(0.12 \mathrm{~mL})$ was added to a growth solution containing CTAB $(50 \mathrm{~mL}, 100 \mathrm{mM}), \mathrm{HAuCl}_{4}(0.5 \mathrm{~mL}, 50 \mathrm{mM})$, ascorbic acid (0.4 mL, $100 \mathrm{mM}), \mathrm{AgNO}_{3}(0.6 \mathrm{~mL}, 10 \mathrm{mM})$ and $\mathrm{HCl}(0.95 \mathrm{~mL}$, $1000 \mathrm{mM})$. The mixture was left undisturbed at $30^{\circ} \mathrm{C}$ for $2 \mathrm{~h}$. The solution was centrifuged twice (8000 rpm, $30 \mathrm{~min}$ ) and redispersed in CTAB $(100 \mathrm{mM})$ to obtain a final concentration of gold equal to $0.5 \mathrm{mM}$.

Synthesis of gold nanostars. The surfactant-free method was used to prepare gold nanostars. ${ }^{39}$ A solution of gold seeds $(\sim 14 \mathrm{~nm}, 0.5 \mathrm{~mL},[\mathrm{Au}]=0.5 \mathrm{mM})$ prepared by the Turkevich method was added to an aqueous solution (50 mL) containing $\mathrm{HAuCl}_{4}(0.25 \mathrm{mM})$ and $\mathrm{HCl}(1 \mathrm{mM})$, followed by the addition of $\mathrm{AgNO}_{3}(10 \mathrm{mM}, 0.15 \mathrm{~mL})$ and ascorbic acid $(100 \mathrm{mM}, 0.25 \mathrm{~mL})$. To increase the stability of the obtained nanostars, CTAB (10 $\mathrm{mL}, 100 \mathrm{mM})$ was added to the growth solution. Upon synthesis, the solution was centrifuged twice $(4500 \mathrm{rpm}$, $30 \mathrm{~min})$ and redispersed in CTAB $(100 \mathrm{mM})$ to obtain a final concentration of gold equal to $0.5 \mathrm{mM}$.

Platinum reduction on gold nanoparticles. Platinum overgrowth was carried out in the presence of silver ions. ${ }^{40}$ To a dispersion of gold nanoparticles $(10 \mathrm{~mL},[\mathrm{Au}]=0.5 \mathrm{mM})$ was added $\mathrm{AgNO}_{3}(0.04 \mathrm{~mL}, 10 \mathrm{mM})$ and $\mathrm{K}_{2} \mathrm{PtCl}_{4}(0.05 \mathrm{~mL}, 10 \mathrm{mM})$ and the mixture was left for $30 \mathrm{~min}$ at $40{ }^{\circ} \mathrm{C}$ to allow for complexation of the platinum salt with CTAB, followed by the addition of ascorbic acid $(0.1 \mathrm{~mL}, 100 \mathrm{mM})$ maintaining the temperature at $40{ }^{\circ} \mathrm{C}$ for $12 \mathrm{~h}$. Pt coated gold nanoparticles were washed twice by centrifugation and redispersed in water to obtain a final concentration of gold equal to $6 \mathrm{mM}$.

Plasmonic substrates. The as-prepared Pt-coated gold nanoparticles $(0.5 \mathrm{mM}, 50 \mathrm{~mL},[\mathrm{CTAB}]=0.1 \mathrm{M})$ were centrifuged and redispersed in CTAB $(1 \mathrm{mM}, 2 \mathrm{~mL})$. The solution was again centrifuged and redispersed in pure water $(1 \mathrm{~mL})$. Parafilms were used to wrap the edges of the glass substrate $(0.8 \times$ $2.5 \mathrm{~cm})$ to produce a container. The solution of nanoparticles was then placed in the container and evaporated at room temperature for 3-5 days.

Photochemical regeneration of cofactor molecules. The plasmonic substrate was immersed in a photochemical mixture ( $1 \mathrm{~mL}$, in quartz cuvette) consisting of phosphate buffer $(0.1 \mathrm{M}$, $\mathrm{pH} 8), \mathrm{NAD}^{+}(1 \mathrm{mM})$ and TEAOH $(1 \mathrm{M})$. The cuvette was irradiated with visible/infrared light. After two hours the plasmonic substrate was removed from the cuvette and the solution was analyzed by UV-vis spectroscopy. The concentration of NADH was determined based on the absorption band intensity at $340 \mathrm{~nm}$.

Photoelectrochemical oxidation of TEAOH. First, the solutions of gold nanoparticles (bare or Pt-functionalized, $10 \mathrm{~mL}$, $0.5 \mathrm{mM})$ in CTAB (100 $\mathrm{mM})$ were centrifuged twice and redispersed in water to obtain a final concentration of gold 6 $\mathrm{mM}$. Six solutions in total of bare and Pt-functionalized nanoparticles $(18.5 \mu \mathrm{L}, 6 \mathrm{mM})$ were dropcast on the working electrode of the screen-printed carbon electrode and evaporated at room temperature for 12 hours. Prior to the measurements the electrode was covered with a photochemical mixture $(50 \mu \mathrm{L}$, TEAOH (1 M), phosphate buffer (0.1 M)). All experiments were performed in the exact manner: once the open circuit potential reached the steady state $(c a .-0.36 \mathrm{~V})$, the lamp (400-1200 nm, $150 \mathrm{~mW} \mathrm{~cm}^{-2}$ ) was switched on and the corresponding output current was recorded for 10 seconds.

\section{Acknowledgements}

This work was supported by the Spanish Ministry of Economy and Competitiveness (MAT2013-49375-EXP, BIO2014-59741-R) and BBVA Foundation - "Primera convocatoria de ayudas fundación BBVA a investigadores, innovadores y creadores culturales”. D. M. S., J. M. T, and F. O acknowledge funding from the European Regional Development Fund (ERDF) and the Spanish Ministry of Economy and Competitiveness (Projects MAT2014-58201-C2-1-R, MAT2014-58201-C2-2-R), from the ERDF and the Galician Regional Government under agreement for funding the Atlantic Research Center for Information and Communication Technologies (AtlantTIC), and from the ERDF and the Extremadura Regional Government (Junta de Extremadura) under Project IB13185. We thank Dr Luis Yate for assistance with XPS analysis.

\section{Notes and references}

1 S. Gao, K. Ueno and H. Misawa, Acc. Chem. Res., 2011, 44, 251-260.

2 S. C. Warren and E. Thimsen, Energy Environ. Sci., 2012, 5, 5133-5146.

3 S. Linic, P. Christopher and D. B. Ingram, Nat. Mater., 2011, 10, 911-921.

4 J. Y. Park, L. R. Baker and G. A. Somorjai, Chem. Rev., 2015, 115, 2781-2817.

5 S. Linic, P. Christopher, H. Xin and A. Marimuthu, Acc. Chem. Res., 2013, 46, 1890-1899.

6 J. C. Scaiano and K. Stamplecoskie, J. Phys. Chem. Lett., 2013, 4, 1177-1187.

7 M. Xiao, R. Jiang, F. Wang, C. Fang, J. Wang and J. Yu, J. Mater. Chem. A, 2013, 1, 5790-5805.

8 C. Wang and D. Astruc, Chem. Soc. Rev., 2014, 43, 7188-7216.

9 C. Clavero, Nat. Photonics, 2014, 8, 95-103.

10 M. J. Kale, T. Avanesian and P. Christopher, ACS Catal., 2013, 4, 116-128.

11 G. Baffou and R. Quidant, Chem. Soc. Rev., 2014, 43, 38983907.

12 M. L. Brongersma, N. J. Halas and P. Nordlander, Nat. Nanotechnol., 2015, 10, 25-34.

13 J. G. Smith, J. A. Faucheaux and P. K. Jain, Nano Today, 2015, 10, 67-80.

14 R. Long, Y. Li, L. Song and Y. Xiong, Small, 2015, 11, 38733889. 
15 P. Zhang, T. Wang and J. Gong, Adv. Mater., 2015, 27, 53285342.

16 S. Mubeen, J. Lee, N. Singh, S. Krämer, G. D. Stucky and M. Moskovits, Nat. Nanotechnol., 2013, 8, 247-251.

17 Z. Zheng, T. Tachikawa and T. Majima, J. Am. Chem. Soc., 2014, 136, 6870-6873.

18 F. Wang, C. Li, H. Chen, R. Jiang, L.-D. Sun, Q. Li, J. Wang, J. C. Yu and C.-H. Yan, J. Am. Chem. Soc., 2013, 135, 55885601.

19 T. T. Trinh, R. Sato, M. Sakamoto, Y. Fujiyoshi, M. Haruta, H. Kurata and T. Teranishi, Nanoscale, 2015, 7, 12435-12444. 20 X. Huang, Y. Li, Y. Chen, H. Zhou, X. Duan and Y. Huang, Angew. Chem., Int. Ed., 2013, 52, 6063-6067.

21 G. L. Hallett-Tapley, M. J. Silvero, C. J. Bueno-Alejo, M. González-Béjar, C. D. McTiernan, M. Grenier, J. C. Netto-Ferreira and J. C. Scaiano, J. Phys. Chem. C, 2013, 117, 12279-12288.

22 W. Xie and S. Schlücker, Nat. Commun., 2015, 6, 7570.

23 L. M. Liz-Marzán, Langmuir, 2006, 22, 32-41.

24 M. Grzelczak and L. M. Liz-Marzán, Langmuir, 2013, 29, 4652-4663.

25 M. Moskovits, Nat. Nanotechnol., 2015, 10, 6-8.

26 R. Long, Z. Rao, K. Mao, Y. Li, C. Zhang, Q. Liu, C. Wang, Z.-Y. Li, X. Wu and Y. Xiong, Angew. Chem., Int. Ed., 2015, 54, 2425-2430.

27 A. O. Govorov, H. Zhang and Y. K. Gun'ko, J. Phys. Chem. C, 2013, 117, 16616-16631.

28 A. O. Govorov, H. Zhang, H. V. Demir and Y. K. Gun'ko, Nano Today, 2014, 9, 85-101.

29 A. O. Govorov and H. Zhang, J. Phys. Chem. C, 2015, 119, 6181-6194.

30 C. S. Kumarasinghe, M. Premaratne, Q. Bao and G. P. Agrawal, Sci. Rep., 2015, 5, 12140.

31 A. Guerrero-Martínez, S. Barbosa, I. Pastoriza-Santos and L. M. Liz-Marzán, Curr. Opin. Colloid Interface Sci., 2011, 16, 118-127.

32 S. Kumar, et al., Nanotechnology, 2008, 19, 015606.

33 A. Sánchez-Iglesias, A. Chuvilin and M. Grzelczak, Chem. Commun., 2015, 51, 5330-5333.

34 Z. Zheng, T. Tachikawa and T. Majima, J. Am. Chem. Soc., 2014, 137, 948-957.
35 C. Kim, Y. Kwon and H. Lee, Chem. Commun., 2015, 51, 12316-12319.

36 L. Weng, H. Zhang, A. O. Govorov and M. Ouyang, Nat. Commun., 2014, 5, 4792.

37 J. Zhang, M. R. Langille, M. L. Personick, K. Zhang, S. Li and C. A. Mirkin, J. Am. Chem. Soc., 2010, 132, 14012-14014.

38 M. Liu and P. Guyot-Sionnest, J. Phys. Chem. B, 2005, 109, 22192-22200.

39 H. Yuan, C. G. Khoury, H. Hwang, C. M. Wilson, G. A. Grant and T. Vo-Dinh, Nanotechnology, 2012, 23, 075102.

40 M. Grzelczak, J. Pérez-Juste, B. Rodriguez-Gonzalez and L. Liz-Marzán, J. Mater. Chem., 2006, 16, 3946-3951.

41 M. R. Langille, J. Zhang, M. L. Personick, S. Li and C. A. Mirkin, Science, 2012, 337, 954-957.

42 D. M. Solís, J. M. Taboada, F. Obelleiro, L. M. Liz-Marzán and F. J. García de Abajo, ACS Nano, 2014, 8, 7559-7570.

43 C. Hamon, S. M. Novikov, L. Scarabelli, D. M. Solís, T. Altantzis, S. Bals, J. M. Taboada, F. Obelleiro and L. M. Liz-Marzán, ACS Photonics, 2015, 2, 1482-1488.

44 M. Grzelczak, J. Pérez-Juste, F. J. García de Abajo and L. M. Liz-Marzán, J. Phys. Chem. C, 2007, 111, 6183-6188.

45 H.-J. Jang, S. Hong, S. Ham, K. L. Shuford and S. Park, Nanoscale, 2014, 6, 7339-7345.

46 C. Novo, A. M. Funston and P. Mulvaney, Nat. Nanotechnol., 2008, 3, 598-602.

47 S. Horikoshi, N. Watanabe, M. Mukae, H. Hidaka and N. Serpone, New J. Chem., 2001, 25, 999-1005.

48 J. Lee, S. Mubeen, X. Ji, G. D. Stucky and M. Moskovits, Nano Lett., 2012, 12, 5014-5019.

49 H. Yang, L.-Q. He, Y.-W. Hu, X. Lu, G.-R. Li, B. Liu, B. Ren, Y. Tong and P.-P. Fang, Angew. Chem., Int. Ed., 2015, 127, 11624-11628.

50 D. M. Solís, J. M. Taboada and F. O. Basteiro, IEEE Trans. Antennas Propag., 2015, 63, 2141-2152.

51 J. M. Taboada, M. G. Araújo, F. O. Basteiro, J. L. Rodriguez and L. Landesa, Proc. IEEE, 2013, 101, 350-363.

52 J. Song, C.-C. Lu and W. C. Chew, IEEE Trans. Antennas Propag., 1997, 45, 1488-1493.

53 D. M. Solís, M. G. Araújo, L. Landesa, S. García, J. M. Taboada and F. Obelleiro, IEEE Photonics J., 2015, 7, 1-9. 